\title{
Combined FEC-PET/MRI improves diagnostic accuracy
}

New data show that combined [18F]fluoroethylcholine-(FEC)-PET/ MRI improves the accuracy of prostate cancer detection compared with PET or MRI alone, and has a very high sensitivity (95\%) for detecting the dominant malignant lesion of the prostate.

In a study of 128 prostate lesions, patients with positive needle biopsy findings were assessed by FEC-PET and MRI in identical positioning, prior to radical prostatectomy. After surgery, these imaging results were compared with histological whole-mount sections, and PET/MRI lesion-based semiquantitative FEC uptake was compared with Gleason scores and postoperative histology.

A main aim of the study was to improve metabolic imaging with FEC-PET by adding only morphological information from MRI, substantially reducing the total scanning time of patients, as well as the number of false positive findings. A second aim was to improve the assessment of tumour aggressiveness by studying semiquantitative FEC-uptake.
Data analysis revealed a sensitivity, specificity, positive predictive value (PPV), negative predictive value, and accuracy of $67 \%, 35 \%, 59 \%, 44 \%$, and $54 \%$, respectively, for MRI versus $85 \%, 45 \%$, $68 \%, 69 \%$, and $68 \%$, respectively, for PET, which increased to $84 \%, 80 \%, 85 \%$, $78 \%$, and $82 \%$, respectively, for combined FEC-PET/MRI of lesions $>5 \mathrm{~mm}(n=98)$. The superiority of the combined approach for detecting lesions $>5 \mathrm{~mm}$ is an important finding, as the clinical relevance of tumours below this threshold size has been called into question in recent years.

\section{《This approach promises to enhance the success rate of needle biopsies... 77}

For lesions in patients with Gleason $>6$ tumours $(n=43)$, these values were $73 \%, 31 \%, 71 \%, 33 \%$, and $60 \%$ for MRI, $90 \%, 62 \%, 84 \%, 73 \%$, and $81 \%$ for PET, and $87 \%, 92 \%, 96 \%, 75 \%$, and $88 \%$ for FEC-PET/MRI. By studying semiquantitative FEC uptake in PET/MRIdefined lesions, cancers with Gleason scores $>6$ were distinguished from those with Gleason score $\leq 6$ with a specificity of $90 \%$ and a PPV of $83 \%$, compared with $71 \%$ and $60 \%$, respectively, for needle biopsy, suggesting a potential role in patient selection for active surveillance and watchful waiting.

"This is the first registered prospective investigator-initiated diagnostic trial of the use of combined FEC-PET/ MRI in men with prostate cancer," says Markus Hartenbach, who led the study. "This approach promises to enhance the success rate of needle biopsies and could improve patient-and-risk adapted treatment, or 'no treatment', strategies in the future. FEC-PET/MRI should be part of further randomized clinical trials."

\section{Melanie Clyne}

Original article Hartenbach, M. et al. Combined PET/MRI
improves diagnostic accuracy in patients with prostate
cancer: A prospective diagnostic trial. Clin. Cancer Res.
doi:10.1158/1078-0432.CCR-13-2653

